\title{
LA INTERNACIONALIZACIÓN DE LA EDUCACIÓN SUPERIOR, FACTOR CLAVE PARA FORTALECER LA CALIDAD EDUCATIVA Y MEJORAR LAS CONDICIONES DE VIDA DE LA SOCIEDAD.
}

\section{INTERNATIONALIZATION OF HIGHER EDUCATION, KEY FACTOR TO ENHANCE EDUCATIONAL QUALITY AND IMPROVE THE LIVING CONDITIONS OF SOCIETY.}

\author{
Jesús Salvador Moncada Cerón \\ Maestría en Educación por la Universidad La Salle, \\ Dr. en Derecho por la Universidad Pontificia, \\ Doctorado en Educación por la Universidad La Salle México. \\ smoncada18@yahoo.com.mx
}

\section{Resumen}

La internacionalización de la educación superior se ha convertido en una dinámica novedosa que ha de integrarse a las funciones clásicas de las instituciones educativas, en afinidad a un conocimiento sin fronteras que se desarrolla en el escenario de la globalización. Al incorporar la dimensión internacional e intercultural se promueve un incremento en la calidad educativa, a la vez que impone retos a la estructura y gestión universitaria respecto a sus problemáticas en torno a la investigación, la docencia, el servicio a la comunidad y la autonomía. Al considerar tales desafíos, la clave de una nueva universidad deberá basarse en el desarrollo humano sustentable, la cultura de paz, la solidaridad y una educación permanente, todo ello, a partir de una transformación significativa del sistema de educación superior, soporte estratégico del desarrollo.

Palabras clave: desarrollo, educación superior, globalización, internacionalización, solidaridad.

\section{Abstract}

The internationalization of higher education has become a new dynamic to be integrated into the traditional functions of educational institutions in affinity to a borderless knowledge on the stage of globalization. By incorporating an international and intercultural dimension we promote an increase in educational quality, as well as challenges imposed to the university management and structure regarding their issues around research, teaching, community service and autonomy. Considering these challenges, the key to a new university should be based on sustainable human development, culture of peace, solidarity and lifelong 
education, all this from a significant transformation of the higher education system, strategic development support.

Keywords: development, higher education, globalization, internationalization, solidarity

\section{Introducción}

La globalización económica ha marcado desde la última década del siglo pasado un modo particular de producir, comercializar y consumir en todos los ámbitos del desenvolvimiento social. La educación ha sido especialmente impactada por esta nueva dinámica y los tratados de liberalización han impuesto la necesidad de internacionalizar el servicio educativo, aspecto sobre el cual se han centrado las inquietudes de los diversos actores que intervienen en este servicio, básicamente en el nivel superior. Al destacar el carácter universal de la investigación y el aprendizaje, la internacionalización se convierte en un planteamiento de ineludible análisis; su consideración deberá, entonces, ser afín a los requerimientos de la solidaridad, del desarrollo, de la equidad y de la paz.

En este contexto, el valor agregado que ofrecen la investigación y la innovación determinan la competitividad de una economía. La economía del conocimiento se construye sobre la capacidad que se tenga de incorporar el conocimiento en todos los sectores del aparato productivo.

La cooperación internacional entre universidades es un elemento intrínseco en los procesos de generación del conocimiento científico y del desarrollo humano que apuntan a la innovación. Lo que hoy precisa la universidad es estructurar planes de desarrollo que establezcan prioridades institucionales y académicas que redimensionen el valor de sus atributos primigenios, vinculados a las nuevas representaciones sociales y a lo que exigen nuestras sociedades absolutamente interdependientes. La internacionalización solidaria se concibe como bien público y derecho social, por lo que ha de estructurarse bajo los principios de la reciprocidad y del respeto a la diversidad (Gazzola, 2007). El papel que hoy juega la universidad consiste en estrechar los vínculos entre los universitarios de los diversos países, para contrarrestar los particularismos y fomentar la ayuda entre los pueblos, mediante una acción metódica al servicio de la ciencia y la cultura. Se hace necesario buscar la comunión de los espíritus en un ambiente de cultura característico que busque la unificación del saber.

\section{La universidad y la internacionalización}

La universidad, por sus características, misión y funciones, representa la conciencia crítica que desde su origen ha contribuido al desarrollo de la herencia cultural de los pueblos. Ella es el espacio ideal en el que sus distintos estamentos necesitan interactuar para lograr una comprensión más profunda sobre lo que es el ser humano, su función social y su fin trascendente. El quehacer universitario se fundamenta en la sociedad, en la solución de sus problemas, en la formación de ciudadanos del mundo y en la incesante búsqueda del conocimiento. 
El escenario actual en el que se desenvuelve la universidad supone retos particulares para la institución. Así, la integración de los bloques mundiales, producto de los procesos de globalización, a través de la cual la población del mundo se ha subordinado a la influencia de un sistema económico, cultural y político centrado en el núcleo de los países más ricos, se ha orientado a la modificación drástica de aspectos de producción y mercado. Tal integración, que ha motivado las cada vez más severas desigualdades económicas, ha impactado en la naturaleza de las relaciones sociales y políticas, en la movilidad geográfica de las personas, cuyo carácter tiene profundas implicaciones en las reconfiguraciones de los ámbitos naturales y sociales, y sin duda, en la estructura de las instituciones, en especial, las de educación superior.

En nuestras sociedades contemporáneas, cada vez más interdependientes, la universidad, como institución emblemática de la educación superior, de la investigación humanística, social y científica, de la creación artística y de la difusión de la cultura, se coloca en el epicentro de la atención social, del debate intelectual, de las preocupaciones políticas y económicas de nuestros días. Hoy procede hablar sobre cuál es el futuro de la universidad y cómo se constituirán las universidades del futuro (De la Fuente, 2007).

Un concepto clave para entender el contexto de la institución universitaria es el de la sociedad del conocimiento. Fabricar y prestar un servicio pasa inevitablemente por la capacidad que se tenga de hacerlo con el valor que se deriva de la tecnología. Así, el valor agregado que ofrecen la investigación y la innovación determinan la competitividad de una economía. La economía del conocimiento se construye, entonces, sobre la capacidad que se tenga de incorporar el conocimiento a todos los sectores del aparato productivo (De la Fuente, 2007).

La reinvención de la universidad, que conlleva una renovación de sus estructuras y tareas, es uno de los grandes desafíos que deben asumir las comunidades académicas para posicionar a la institución universitaria como la entidad generadora del verdadero desarrollo humano y social en nuestro tiempo. Afín a las condiciones que imponen los grandes paradigmas del siglo XXI: el desarrollo humano sustentable, la cultura de paz, la solidaridad y la educación permanente, la universidad necesita ser el agente de un cambio constructivo que apuntale su compromiso con los procesos de innovación. La internacionalización de la educación superior es factor clave para incrementar la calidad educativa y para formar profesionales capaces de adaptarse y actuar con eficacia en el escenario internacional. La internacionalización surge, así como una respuesta al fenómeno de la globalización, en donde la institución universitaria reivindica su papel de participante activo en la sociedad del conocimiento. Knight (2005) ilustra el impacto de la globalización en la internacionalización de la educación superior:

\begin{tabular}{|l|l|l|}
\hline $\begin{array}{l}\text { Elementos de la } \\
\text { globalización }\end{array}$ & $\begin{array}{l}\text { Impacto en la } \\
\text { educación superior }\end{array}$ & $\begin{array}{l}\text { Consecuencias para la } \\
\text { dimensión internacional } \\
\text { de la educación } \\
\text { superior }\end{array}$ \\
\hline $\begin{array}{l}\text { Sociedad del } \\
\text { conocimiento } \\
\text { Creciente importancia }\end{array}$ & $\begin{array}{l}\text { El creciente énfasis en } \\
\text { educación continuada, } \\
\text { aprendizaje permanente, }\end{array}$ & $\begin{array}{l}\text { Nuevos tipos de } \\
\text { educadores } \\
\text { (compañías privadas de }\end{array}$ \\
\hline
\end{tabular}




\begin{tabular}{|c|c|c|}
\hline $\begin{array}{l}\text { Tecnologías de } \\
\text { información y } \\
\text { comunicación } \\
\text { Nuevos desarrollos de } \\
\text { ecnologías y sistemas de } \\
\text { comunicación e } \\
\text { nformación. }\end{array}$ & $\begin{array}{l}\text { La necesidad de } \\
\text { desarrollar nuevos } \\
\text { conocimientos y } \\
\text { experiencia está dando } \\
\text { como resultado nuevos } \\
\text { tipos de programas y } \\
\text { calificaciones. } \\
\\
\text { El papel de las } \\
\text { universidades en la } \\
\text { producción de } \\
\text { investigación y } \\
\text { conocimiento está } \\
\text { cambiando y está siendo } \\
\text { más comercializado. }\end{array}$ & 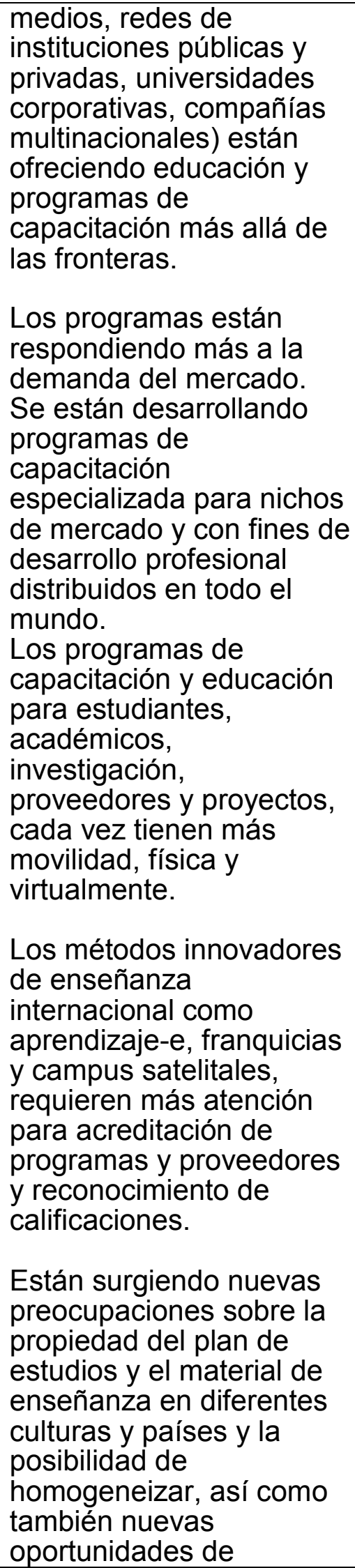 \\
\hline
\end{tabular}




\begin{tabular}{|c|c|c|}
\hline $\begin{array}{l}\text { disminuir barreras } \\
\text { comerciales }\end{array}$ & eliminan barreras. & cruzamientos. \\
\hline $\begin{array}{l}\text { Buen gobierno } \\
\text { Creación de nuevas } \\
\text { estructuras y sistemas de } \\
\text { buen gobierno regional e } \\
\text { internacional. }\end{array}$ & $\begin{array}{l}\text { El papel de los actores a } \\
\text { nivel de educación } \\
\text { nacional, gubernamental } \\
\text { y no gubernamental, está } \\
\text { cambiando. } \\
\text { Se están considerando } \\
\text { en todos los niveles } \\
\text { nuevas estructuras } \\
\text { reguladoras y de } \\
\text { políticas. }\end{array}$ & $\begin{array}{l}\text { Se está dando más } \\
\text { énfasis a los programas } \\
\text { educativos de } \\
\text { importaciones y } \\
\text { exportaciones } \\
\text { comercialmente } \\
\text { orientadas y menos a los } \\
\text { proyectos internacionales } \\
\text { de desarrollo. } \\
\text { Se están considerando } \\
\text { nuevas estructuras } \\
\text { internacionales y } \\
\text { regionales para } \\
\text { complementar políticas y } \\
\text { prácticas nacionales y } \\
\text { regionales especialmente } \\
\text { en el campo del } \\
\text { aseguramiento de } \\
\text { calidad, acreditación, } \\
\text { transferencia de créditos, } \\
\text { reconocimiento de } \\
\text { calificaciones y movilidad } \\
\text { de estudiantes. }\end{array}$ \\
\hline
\end{tabular}

Fuente: Knight (2004), citado por Knight et al (2005).

La internacionalización implica infundir entre los estudiantes, el personal académico y administrativo, nuevos conocimientos, habilidades y actitudes que les permitan funcionar de manera eficaz en un medio internacional y multicultural. Para esto, plantea la integración de la dimensión internacional en la docencia, la investigación, la extensión, en la movilidad académica y estudiantil, en la colaboración investigadora, en la asistencia técnica y en la ayuda para el desarrollo, entre otros rubros.

Para comprender la relevancia de la internacionalización, la UNESCO estableció en 1995 que el diseño de políticas para el cambio y el desarrollo en la educación superior, debía contemplar a la internacionalización dado el carácter universal del aprendizaje y la investigación.

1. En este marco la UNESCO planteó que:

2. La internacionalización se ve fortalecida por los actuales procesos de integración económica y política y por la creciente necesidad de entendimiento intercultural.

3. Es evidente la considerable expansión de diversos tipos de redes y otros mecanismos de enlace entre instituciones, profesores y estudiantes la cual, se ve facilitada por el progreso constante de las tecnologías de información y comunicación. 
4. La cooperación internacional debe basarse ante todo, en la asociación y la búsqueda colectiva de la calidad y la pertinencia.

5. Las condiciones adversas en que funcionan las Instituciones de Educación Superior (IES), en especial en algunos países en desarrollo, deben ser corregidas en función de la solidaridad internacional.

6. Es importante promover programas e intercambios que permitan reducir los desequilibrios existentes y facilitar el acceso a los conocimientos y su transferencia.

La UNESCO ha señalado la importancia de estimular la movilidad académica y profesional con el fin de favorecer el proceso de integración económica, educativa, política y cultural, para fortalecer los proyectos de cooperación horizontal, la creación de nuevas redes universitarias, redes de posgrado y las instancias de intercambio. Asimismo, la OCDE propone aplicar las estrategias de internacionalización a tres niveles diferentes: micro (el proceso de enseñanza aprendizaje en el salón de clase), medio (el plan de estudios) y macro (políticas definitivas y estrategias institucionales). En este último rubro, es necesario entender que la internacionalización es un concepto que precisa de políticas y estrategias institucionales específicas, las que permitirán que se supere el retraso de ciertas regiones, particularmente la latinoamericana, por medio de alianzas estratégicas que suministren a los estudiantes competencias interculturales y globales.

Al trabajar en torno a la internacionalización se fomenta el desarrollo de grupos calificados de investigación, se promueven trabajos sobre temas de interés multilateral; se establecen puentes para el intercambio de recursos humanos, se dan las condiciones para formar docentes e investigadores del más alto nivel, se generan vínculos entre pares de la comunidad y se posibilita la aproximación a centros de excelencia en el mundo entero. De igual forma, se propone la integración de la dimensión internacional en la docencia, la investigación, la extensión; en la movilidad académica y estudiantil, en la colaboración investigadora, en la asistencia técnica y en la ayuda para el desarrollo, entre otros aspectos.

Algunas de las estrategias más consistentes para avanzar en el desarrollo de los mecanismos de internacionalización de las IES son: Incrementar el número de estudiantes, profesores e investigadores en esquemas de movilidad académica, plantear el crecimiento del número de carreras, programas y diplomas enfocados en temas comparativos e internacionales, dar más énfasis al desarrollo de competencias internacionales, interculturales y globales; generar mayor interés en asuntos internacionales y en la investigación colectiva, fomentar el crecimiento de la oferta transfronteriza de programas académicos, desarrollar nuevas redes internacionales y de consorcios; aumentar la cantidad de actividades extracurriculares centradas en el campus con un componente internacional o multicultural, aumentar los programas de doctorado conjuntos o dobles, y establecer nuevas organizaciones nacionales, regionales e internacionales cuyo interés principal sea la educación internacional. En cualquier caso, las propuestas 
de internacionalización necesitan responder a la solución de demandas particulares y globales de orden social, y en cuyo proceso se exalte la parte ética, solidaria y humanista, y en la que se preserven las peculiaridades identitarias de cada cultura.

Los procesos de internacionalización, ineludiblemente necesarios por su potencial constructivo, también se enfrentan a circunstancias contextuales que aún siguen imperando y que se convierten en problemas estructurales, tales como la falta de confianza recíproca entre países y la incapacidad técnica para establecer o legitimar equivalencias, de créditos, a nivel nacional o internacional siendo alto el riesgo de que el reconocimiento y la revalidación conformen un cuello de botella, así como la existencia de diferencias torales que separan los sistemas de educación superior en el país y de éste en relación con las demás naciones. Sin embargo, es preciso entender que los sistemas nacionales no pueden existir de manera aislada.

Hay aspectos que emergen en la esfera internacional y éstos son el desconcierto, el temor y una profunda inseguridad social, circunstancias que desaceleran la movilidad mundial. Su expresión más abierta es la restricción que imponen los países del Primer Mundo para la expedición de visas a nacionales de países en desarrollo. La inquietud que se manifiesta en el imaginario internacional respecto a la "amenaza terrorista" y la galopante extensión del crimen organizado, por la vía de los países emergentes, aunada a la actual crisis económica, la más grave desde 1929, -que ha creado desplazamientos desordenados-, han propiciado medidas extraordinariamente retardatarias. Cada vez es más problemático que el ciudadano de un país en desarrollo ingrese, bajo cualquier necesidad, a una nación poderosa. Somos testigos de una peculiar paradoja en la que se exige la apertura, pero se cierran los cauces para la libre movilidad. En este sentido, se da un profundo revés al avance de los procesos de internacionalización, si bien es incontrovertible que su desarrollo es vital para la comunidad global.

\section{La internacionalización en los países con economías emergentes}

El Informe del Programa de las Naciones Unidas para el Desarrollo (PNUD) sobre el Desarrollo Humano correspondiente al año 1999, señala que es necesario pasar de la globalización neoliberal de los mercados, a la globalización de la sociedad. Ya no es posible acumular sólo utilidades, se necesita globalización con ética, equidad, inclusión, solidaridad, seguridad, sostenibilidad y desarrollo humano. Hasta el momento, la globalización neoliberal ha polarizado a la población. Del Producto Interno Bruto Mundial casi el 80 por ciento corresponde a los países industrializados y sólo un 20 por ciento a los países en desarrollo.

América Latina, según el Banco Mundial, es el continente que presenta la más extrema polarización económica: el 20 por ciento de la población más pobre apenas recibe el 4 por ciento del ingreso total, mientras que el 10 por ciento de la población concentra el 60 por ciento del ingreso. 
Las regiones que aspiren a competir en los nuevos espacios económicos tienen que dar atención prioritaria a la formación de sus recursos humanos del más alto nivel, al desarrollo científico, al progreso técnico y a la acumulación de información, todo lo cual significa apuntalar las inversiones en educación, ciencia, tecnología e investigación. Frente a estos desafíos, la universidad tiene un papel preponderante como promotor de desarrollo. Su tarea debe iniciar con la flexibilización de su estructura académica e introducir el paradigma del aprendizaje permanente, patrocinar sólidos y amplios programas de actualización y superación académica, acompañados de estímulos laborales; incorporarse a la cultura de pertinencia, de calidad, de evaluación, de informática, de administración estratégica y de internacionalización, todo fundamentado en una dimensión ética y de rendición social de cuentas. La educación superior, de cara al siglo XXI, debe asumir el cambio, lo que exige una disposición a la reforma de sus estructuras y métodos de trabajo. La incorporación de una visión prospectiva hará posible que las universidades contribuyan a la elaboración de proyectos futuros de sociedad, inspirados en la solidaridad, en la equidad y en la protección ecológica.

La globalización y sus consecuencias tienen que formar parte central de la reflexión y el análisis de la universidad latinoamericana. Su mirada minuciosa y objetiva deberá prever escenarios futuros con el fin de construir desde ahora las condiciones que den lugar al escenario más favorable para nuestros países. Su objetivo es contribuir al diseño de verdaderos proyectos de nación que al insertarse favorablemente en el contexto internacional, influyan en el desarrollo de una globalización capaz de superar el paradigma neoliberal imperante.

\section{La movilidad internacional}

La trayectoria de la internacionalización, al ser impulsada de manera vertical, ha propiciado una distribución desigual de ventajas, concentrada en pequeños grupos.

De acuerdo a la OCDE (2008), la movilidad ha manifestado los siguientes rasgos:

- Hay una fuerte tendencia hacia la internacionalización de la educación superior que se observa con un crecimiento sin precedentes en las últimas tres décadas pues se pasó de 0.6 millones de estudiantes extranjeros a 2.9 millones en el año 2006.

- Hay una tendencia proactiva por parte de los países del Sudeste Asiático para estimular su internacionalización en materia de educación superior, por el contrario Estados Unidos, que ha sido el destino tradicional de estudiantes extranjeros, mantiene una actitud pasiva y receptiva. Cabe destacar que el dominio de la lengua inglesa sigue siendo un factor determinante en la elección de un destino académico.

- En los países de la OCDE y algunos países asociados, la proporción de estudiantes internacionales que viajan a un país extranjero va de $1 \%$ a $18 \%$ de la matrícula. 
- Los países que se están convirtiendo de manera acelerada en polos de atracción de un número mayor de estudiantes extranjeros son Australia, Austria, Nueva Zelanda, Suiza y el Reino Unido. Japón tiene la misma capacidad de atraer estudiantes provenientes de Asia.

- Desde el año 2000, el número de estudiantes internacionales en educación superior que se inscribieron en países pertenecientes a la OCDE se incrementó en un promedio anual de $7.5 \%$.

- En 2006, más de 2.9 millones de estudiantes de licenciatura se matricularon fuera de sus países de origen; esto representa un $3 \%$ más respecto a 2005 , de acuerdo con los datos reportados por la OCDE y por el Instituto de Estadística de la UNESCO.

- Cabe destacar que de los 2.9 millones, el 83.5\% (2.4 millones), lo hacen en países que pertenecen a la OCDE.

- Entre 2000 y 2006 en promedio, el número de estudiantes extranjeros creció más rápido en los países de la OCDE que en 19 de los países de la Unión Europea.

- Del año 2000 a 2008 duplicaron el número de estudiantes extranjeros la República Checa, Corea, los Países Bajos, Nueva Zelanda, España y Estonia. Si bien en Estados Unidos se concentraba el $20 \%$ de la matrícula internacional, en este país su crecimiento en el pasado lustro fue de sólo $25 \%$ o menos.

- En 2006 Francia (8\%), Alemania (9\%), el Reino Unido (11\%) y los Estados Unidos (20\%) recibieron al $49 \%$ de estudiantes extranjeros de todo el mundo. Pero en términos absolutos, el mayor número de estudiantes internacionales de los países de la OCDE son Francia, Alemania, Japón y Corea. China y la India ocupan un lugar intermedio en el número de estudiantes que envían a países asociados.

- Más del $20 \%$ de los estudiantes internacionales interesados en programas avanzados de investigación, se encuentran inscritos en Bélgica, Canadá, Nueva Zelanda, Suiza, el Reino Unido y los Estados Unidos.

- Alemania, Nueva Zelanda, Suiza y Estados Unidos son los países que atraen a estudiantes interesados en las ciencias duras. Finlandia, Canadá, Alemania, Hungría, Suecia, Suiza, el Reino Unido y Estados Unidos son países que resultan de alto interés a estudiantes de agricultura, ingeniería y ciencias. Los estudiantes interesados en humanidades y artes eligen Austria, Alemania, Japón, Noruega y Eslovenia. Las ciencias sociales y el derecho tienen como polos de atracción Australia, Nueva Zelanda, los Países Bajos, el Reino Unido, Francia y Portugal. Por último, son centros de atracción para el estudio de las ciencias de la salud Bélgica, Dinamarca, Hungría, Italia, Polonia y España.

- El 30\% o más de los estudiantes internacionales están matriculados en ciencias, agricultura o ingeniería en Finlandia, Alemania, Hungría, Suecia, Suiza y Estados Unidos.

- Respecto de la matrícula total por país es importante observar que el $17.8 \%$ en Australia son extranjeros; en Austria el $15.5 \%$ son extranjeros; y en Nueva Zelanda, Suecia y el Reino Unido el $15.5 \%, 13.7 \%$ y $14 \%$ respectivamente, provienen de otros países. En sentido opuesto, menos del $1 \%$ de la matrícula de Corea, Polonia, Turquía y la Federación Rusa son estudiantes extranjeros. 
- La oferta internacional que se proporciona con dispositivos electrónicos y que incluye programas flexibles en campos virtuales ubicados en diversos países ha aumentado su relevancia; sin embargo, no hay datos que permitan evaluar su impacto.

- Los estudiantes asiáticos constituyen el grupo más grande que se inscribe en un país extranjero (representan el $45.3 \%$ de la movilidad estudiantil) y prefieren Australia, Japón, Corea y Nueva Zelanda como país de destino. En los países de la OCDE, $23 \%$ de los estudiantes de origen europeo constituyen el segundo grupo que decide realizar estudios en el extranjero; el 9.9\% son africanos; el 3.5 son norteamericanos y el $5 \%$ pertenecen a Sudamérica.

- China es el país que más estudiantes envía al extranjero; en 2006 los estudiantes chinos representaron el $15.4 \%$ del total matriculados en los países de la OCDE. Estos estudiantes eligen fundamentalmente a Estados Unidos y a Japón como destinos académicos. Después de los chinos, los países que más estimulan la movilidad son India (5.4\%), Marruecos (1.6\%), Malasia (1.6\%) y la Federación Rusa (1.2\%).

- Las razones por las que los estudiantes eligen un país para realizar estudios en el extranjero son múltiples, entre ellas destacan: la lengua (inglés, francés y alemán); el prestigio académico de instituciones o programas; la flexibilidad de los programas, el tiempo que se requiere para obtener un grado académico; la falta de infraestructura, el limitado desarrollo o la carencia de un campo específico en el país de origen; los vínculos históricos entre países; la percepción acerca de las oportunidades laborales en un país extranjero; las aspiraciones culturales y las políticas gubernamentales que facilitan la transferencia de créditos entre instituciones.

\section{La transnacionalización educativa}

La transnacionalización de las instituciones de educación superior ha vulnerado el carácter público de la educación y ha puesto en jaque a los gobiernos, quienes a pesar de los riesgos, carecen de bases que regulen y normen la presencia de entidades extranjeras. Los déficits de regulación propician que los organismos de control o de acreditación tengan dificultad para ejercer y respetar sus atribuciones. De este modo, el aseguramiento de la calidad es otro problema, pues las acreditaciones que proporcionan agencias internacionales no son fiables en muchos casos. Emerge así un mercado internacional de aseguramiento de la calidad que da pocas certezas y en el cual América Latina participa como consumidor, más que como proveedor.

En este ámbito regional de América Latina y el Caribe también se presentan dificultades que se centran en la problemática homologación de los títulos nacionales con los extranjeros. Entre otras razones, lo anterior se debe a la caducidad de tratados de reciprocidad, a los complicados procesos administrativos en varios países; a la carencia de una normatividad internacional específica que reconozca los títulos, grados y cédulas para el ejercicio profesional en otros 
países; a la falta de perfiles profesionales en los que se determinen competencias, así como la ausencia de los criterios de calidad para la acreditación de programas. De igual modo, la ausencia de un plan regional que trascienda los intereses institucionales aislados se suma, al final, en la falta de políticas regionales e internacionales que respalden sólidamente la internacionalización.

Urge además, valorar la pertinencia de los programas de cooperación en su modalidad solidaria, diversificar la oferta de formación, rediseñar el currículo, procurar fondos, incorporar consolidadamente las nuevas tecnologías, y constituir redes de investigación en escala multilateral.

Otros desafíos que necesitan abordarse es el reconocimiento mutuo de créditos, la transferencia de los títulos, la armonización de los sistemas o la circulación de competencias; la revalidación y el reconocimiento de créditos se están convirtiendo en problemáticas severas que mientras no se resuelvan impedirán que América Latina se desarrolle como receptora de estudiantes extranjeros. No obstante, aunque ésta es una circunstancia imperante, empiezan a crearse instancias como el Mecanismo Experimental de Acreditación del Carreras de Grado del MERCOSUR Educativo, el programa TUNING de cooperación entre universidades de América Latina y la Unión Europea, que impulsan la colaboración institucional para mejorar la calidad, la colaboración, la efectividad y la transparencia; y el programa Seis por Cuatro, propuesto de manera conjunta por el Centro Nacional de Evaluación, en México, (CENEVAL) y el programa COLUMBUS de la Unión Europea. En él se pretende el desarrollo de mecanismos que faciliten el reconocimiento de las calificaciones y competencias en seis profesiones (Administración, Ingeniería Electrónica, Medicina, Química, Historia y Matemáticas) y cuatro ejes (competencias profesionales, créditos académicos, evaluación y acreditación, y formación para la investigación y la innovación).

La migración calificada hacia países desarrollados es otro elemento de difícil cuantificación y solución, pues evidentemente las asimetrías sociales entre Norte y Sur motivan a los académicos para que opten por el estilo, calidad y estabilidad de vida que ofrecen los países desarrollados.

\section{Las funciones de los actores educativos en la internacionalización}

La internacionalización de las universidades con los sectores públicos y privados puede considerarse como una actividad con un alto potencial para generar beneficios para ambas partes. La participación de las instituciones académicas en esta tarea no sólo debe entenderse como un mecanismo adicional para financiar labores de investigación. La colaboración de las universidades debe entenderse como una parte fundamental de su compromiso con la sociedad en su conjunto. Se trata de la obligación de las instituciones de educación superior consistente no sólo en educar a la sociedad, sino en proveerla con los conocimientos necesarios para afrontar los problemas de la vida cotidiana. 
Las relaciones entre los diferentes actores de la vinculación no han estado exentas de problemas y de obstáculos. Algunos autores se han ocupado del estudio de los principales problemas endógenos y exógenos de la internacionalización.

A continuación se mencionan algunos de los problemas endógenos:

- Falta de claridad y precisión sobre el significado y ámbito de acción de las actividades de internacionalización.

- Intrascendencia de las actividades de internacionalización dentro de la planeación de las funciones sustantivas.

- Avance incipiente de las actividades de investigación y desarrollo tecnológico en muchas de las instituciones y, por tanto, de los bienes y servicios que se puedan ofrecer a la sociedad.

- Desarrollo insuficiente de investigación aplicada y de resultados de utilización inmediata.

- Desarticulación entre investigación y docencia, lo que propicia la existencia de currícula no actualizada.

- Resistencia por parte de los investigadores para asumir compromisos docentes.

- Ausencia de práctica profesional dentro de muchos de los programas académicos y, por lo mismo, una deficiente formación de los egresados, lo que dificulta su incorporación al mercado laboral.

- Diferentes culturas, valores y principios entre académicos y empresarios.

- Falta de compatibilidad entre los servicios y productos que ofrecen las instituciones de educación superior y los que demandan los sectores social y productivo.

- Inexistencia de instancias colegiadas que propicien la articulación de las actividades académicas con los requerimientos del sector productivo.

- Entre los problemas exógenos se encuentran:

- Falta de coordinación entre las políticas de ciencia y tecnología y las de fomento industrial, agropecuario y de servicios, de educación y de cooperación internacional, entre otras.

- Insuficiencia de recursos para el desarrollo de actividades de investigación y desarrollo tecnológico.

- Existencia de una secuela nociva en el desarrollo de la investigación científica básica, aplicada y experimental, así como de avances tecnológicos en áreas estratégicas para el desarrollo productivo del país, derivada de un largo periodo de protección industrial.

- Desinterés, desconfianza y desconocimiento del sector empresarial sobre los bienes y servicios que pueden aportar las instituciones de educación superior.

\section{Conclusiones}

La cooperación internacional entre universidades es un elemento intrínseco en los procesos de generación del conocimiento científico y del desarrollo tecnológico que apuntan a la innovación. Asimismo, se comprende la importancia de concebir la internacionalización como factor clave para el desarrollo de grupos calificados 
de investigación y para la promoción de trabajos sobre temas de interés multilateral.

Al trabajar en torno a la internacionalización se establecen puentes para el intercambio de recursos humanos, se pretende formar docentes e investigadores del más alto nivel, se generan vínculos entre pares de la comunidad y se posibilita la aproximación a centros de excelencia en el mundo entero.

La internacionalización es estratégica para elevar la calidad de la educación y para formar profesionales que puedan actuar con eficacia en el escenario internacional. En el proceso, las universidades, con una perspectiva de futuro, deben desempeñar un papel esencial en la creación de cuadros profesionales capaces de adaptarse a la nueva realidad global. No obstante, la tarea no sólo debe centrarse en preparar universitarios de acuerdo a un perfil exigido por el mercado internacional, sino en formar ciudadanos con una educación humanista y con vocación social, que sean capaces de conocer, respetar y apreciar las diferencias culturales de los países para actuar en un mundo que demanda desarrollo, paz y equidad.

Gracias a los esfuerzos de los gobiernos de diversos países de la comunidad Europea, India y China, entre otros, se han conformado sólidos entramados que apuntalan la internacionalización como factor esencial de los procesos académicos. Se ha internacionalizado el currículo, se han movilizado los recursos culturales y económicos entre regiones y se han llevado a cabo importantes iniciativas que promueven la movilidad para alentar el contacto intercultural y la construcción de redes sociales. Sin embargo, en América Latina y el Caribe aún es incipiente el desarrollo de movimientos análogos y subsisten enormes conflictos que limitan la acción internacional. Paradójicamente, la región se ha convertido en receptora de proveedores educativos extranjeros que a través de franquicias, y con la anuencia, e incluso protección, de autoridades civiles y educativas, han lucrado con la educación por sus objetivos básicamente comerciales de dudosa calidad académica. Éste es el riesgo de una apertura que se basa en fines económicos, pero que es ajena a la necesidad ingente de impulsar políticas y estrategias serias de internacionalización orientadas al verdadero desarrollo en nuestros países. El fenómeno es poderoso, por lo quecabría entablar una responsabilidad compartida sustentada en la solidaridad internacional para disminuir la enorme brecha entre países al entender que la internacionalización es un proceso que compromete a la comunidad mundial.

Por lo referido a lo largo de este análisis, es claro que uno de los grandes compromisos por parte de las instituciones de educación superior es reconocer la relevancia de promover y diversificar las acciones encaminadas a la internacionalización. Esto resulta vital para crear nuevas y promisorias oportunidades que permitan vincular a los universitarios con sus pares en el mundo, ello con la expectativa de propiciar sinergias que hagan del esfuerzo mancomunado la tarea esencial que privilegie la cooperación, la solidaridad y la convivencia en una nueva cultura de paz. 


\section{FUENTES DE CONSULTA}

- CINDA, (1998) Cooperación Universitaria- Empresa: Visiones de Europa y América Latina, Santiago, Chile.

- DIDOU, S. (2006) Internacionalización de la educación superior: entre el entusiasmo y el desencanto, en Perfiles Educativos, México: Centro de Estudios sobre la Universidad. Universidad Nacional Autónoma de México.

- FIUC (1984), La Universidad hoy en Latinoamérica, Bogotá.

- FUENTE DE LA, Juan Ramón (2007) Discurso del Rector en la ceremonia de investidura de siete personalidades como Doctores Honoris Causa en la UNAM, México, Dirección General de Comunicación Social de la UNAM. Gaceta UNAM.

- GACEL-AVILA, J. (1999), Internacionalización de la educación superior en América Latina y el Caribe. Reflexiones y lineamientos. OUI, AMPEI, Ford Foundation, Guadalajara: Editorial Gráfica Nueva Occidente.

- GAZZOLA, AL (2007). Prólogo al libro de Didou y cols. Experiencias de convergencia de la educación superior en América Latina, México: UNESCO/CINVESTAV.

- HERRERA, A. (2009), Prólogo al libro de Moncada, JS. La internacionalización de la educación superior. El caso de la Universidad La Salle, en: Reflexiones universitarias, $\mathrm{N}^{\circ} 84$, Universidad La Salle, México.

- KNIGHT, J. y DE WIT, H. (1995), Strategies for internationalisation of higher education: historical and conceptual perspectives, Amsterdam, European Association for International Education / Organization for Economic Cooperation and Development / Association of International Education Administrators.

- KNIGHT, J. y DE WIT, H. (1993), Internationalisation: Management strategies and issue, Ottawa: CBIE

- UNESCO (1998) La educación superior del siglo XXI: Visión y acción. Informe final, París.

- UNESCO (1995). Documento de política para el cambio y el desarrollo de la educación superior. París, Francia

- GATS in the light of increasing internationalisation of higher education. Quality assurance and recognition En línea en: http://www.oecd.org/datoecd/37/10/2751067.pdf

- La educación superior en las negociaciones de comercio internacional: opciones planteadas al espacio regional del MERCOSUR, presentado por 
el presidente de la Comisión Sectorial del MERCOSUR, En línea: www.rau.edu.uy

- (2004)Foro sobre el Comercio de la Educación y el boletín Policy Brief, www.oecd.org

- (1998) La Conferencia Mundial sobre Educación Superior, 5-9 de octubre, En línea en: www.unesco.org

- MERCOSUR educativo, En línea en: http://sicmercosul.mec.gob.br/

- UNESCO, Marco de acción prioritaria para el cambio y el desarrollo de la educación superior, En línea en: http://www.educacion.unesco.org/educprog/wche/presentacion.htm 